\title{
Intraovarian Route of Administration
}

National Cancer Institute

\section{Source}

National Cancer Institute. Intraovarian Route of Administration. NCI Thesaurus. Code C38256.

Administration of a drug within the ovary or ovaries. 